\title{
T2-Imaging of the ischemic area-at-risk predicts recovery of cardiac function after acute ST- elevation myocardial infarction
}

\author{
Jamieson M Bourque ${ }^{1,2^{*}}$, Emily A Pearce ${ }^{2}$, Ashul Govil ${ }^{2}$, Anshul Aggarwal ${ }^{2}$, Amit Bhaskar ${ }^{2}$, Christopher M Kramer ${ }^{1,2}$ \\ From 15th Annual SCMR Scientific Sessions \\ Orlando, FL, USA. 2-5 February 2012
}

\section{Background}

T2-weighted edema imaging identifies the ischemic area at risk during acute myocardial infarction. Myocardial salvage occurs in the area at risk without late gadolinium enhancement (LGE). The degree of myocardial salvage is prognostically important, but its effect on recovery of left ventricular (LV) function is not known. The purpose of this study was to determine if the degree of myocardial salvage predicts recovery of function after an acute ST-elevation myocardial infarction (STEMI).

\section{Methods}

We assessed patients with no known prior CAD postSTEMI. Imaging was performed within 72 hours of the acute event and was then repeated 6-12 weeks postinfarction. The degree of myocardial salvage was obtained by subtracting the percentage LGE from the T2 area at risk. The degree of salvage was compared to the percentage improvement in LVEF by nonparametric Spearman rank correlation coefficient analysis.

\section{Results}

Twenty-three patients were recruited for the study. One patient refused a second study, 2 had technical difficulties, and 5 had a baseline LV ejection fraction (EF) of $\geq 50 \%$, leaving a final study population of 15 subjects. The mean age of the sample was $59.3 \pm 11.7$ years; 86.7\% were male and $93.3 \%$ were Caucasian. Hypertension, hyperlipidemia, and tobacco use was present in $40.0 \%, 73.3 \%$, and $46.7 \%$, respectively. The mean BMI was $30.8 \pm 4.1$, and $9 / 15(60 \%)$ had a $\mathrm{BMI} \geq 30$. The mean LDL was $118.7 \pm 33.0$, and the mean $\mathrm{HgAlc}$ was $6.7 \pm 1.8$. The baseline mean LVEF was $42.1 \pm 6.6 \%$, and increased to $45.1 \pm 9.8 \%$, a mean increase of $3.0 \%$. The mean percentage T2 enhancement was $41.2 \pm$ $17.4 \%$, and the mean burden of LGE was $23.5 \pm 14.0 \%$, giving a percentage myocardial salvage of $17.7 \%$. The degree of myocardial salvage correlated moderately with an improvement of LVEF from the acute to recovery CMR, with a Spearman correlation coefficient of 0.59 $(\mathrm{p}=0.020)$.

\section{Conclusions}

The percentage of myocardial salvage moderately predicts recovery of function after an acute STEMI. Future analysis will focus on identifying additional predictors on post-infarction cardiac magnetic resonance imaging.

\section{Funding}

None.

\section{Author details}

'Radiology, University of Virginia Health System, Charlottesville, VA, USA ${ }^{2}$ Medicine, University of Virginia Health System, Charlottesville, VA, USA.

Published: 1 February 2012

doi:10.1186/1532-429X-14-S1-P26

Cite this article as: Bourque et al.: T2-Imaging of the ischemic area-atrisk predicts recovery of cardiac function after acute ST-elevation myocardial infarction. Journal of Cardiovascular Magnetic Resonance 2012 14(Suppl 1):P26. 